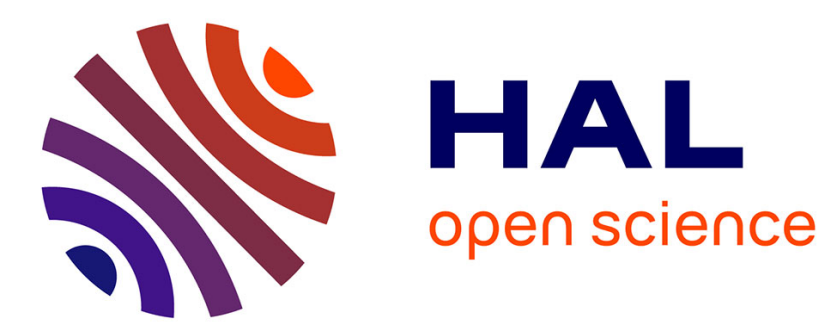

\title{
Bounded Phase Analysis of Message-Passing Programs
}

Ahmed Bouajjani, Michael Emmi

\section{To cite this version:}

Ahmed Bouajjani, Michael Emmi. Bounded Phase Analysis of Message-Passing Programs. 2011. hal-00653085v2

\section{HAL Id: hal-00653085 \\ https://hal.science/hal-00653085v2}

Submitted on 27 Jan 2012

HAL is a multi-disciplinary open access archive for the deposit and dissemination of scientific research documents, whether they are published or not. The documents may come from teaching and research institutions in France or abroad, or from public or private research centers.
L'archive ouverte pluridisciplinaire HAL, est destinée au dépôt et à la diffusion de documents scientifiques de niveau recherche, publiés ou non, émanant des établissements d'enseignement et de recherche français ou étrangers, des laboratoires publics ou privés. 


\title{
Bounded Phase Analysis of Message-Passing Programs*
}

\author{
Ahmed Bouajjani and Michael Emmi ${ }^{\dagger}$ \\ LIAFA, Université Paris Diderot, France \\ $\{$ abou,mje\}@liafa.jussieu.fr
}

\begin{abstract}
We describe a novel technique for bounded analysis of asynchronous message-passing programs with ordered message queues. Our bounding parameter does not limit the number of pending messages, nor the number of "contexts-switches" between processes. Instead, we limit the number of process communication cycles, in which an unbounded number of messages are sent to an unbounded number of processes across an unbounded number of contexts. We show that remarkably, despite the potential for such vast exploration, our bounding scheme gives rise to a simple and efficient program analysis by reduction to sequential programs. As our reduction avoids explicitly representing message queues, our analysis scales irrespectively of queue content and variation.
\end{abstract}

\section{Introduction}

Software is becoming increasingly concurrent: reactivity (e.g., in user interfaces, web servers), parallelization (e.g., in scientific computations), and decentralization (e.g., in web applications) necessitate asynchronous computation. Although sharedmemory implementations are often possible, the burden of preventing unwanted thread interleavings without crippling performance is onerous. Many have instead adopted asynchronous programming models in which processes communicate by posting messages/tasks to others' message/task queues - Miller et al. 18] discuss why such models provide good programming abstractions. Single-process systems such as the JavaScript page-loading engine of modern web browsers [1], and the highly-scalable Node.js asynchronous web server [10], execute a series of short-lived tasks one-by-one, each task potentially queueing additional tasks to be executed later. This programming style ensures that the overall system responds quickly to incoming events (e.g., user input, connection requests). In the multi-process setting, languages such as Erlang and Scala have adopted messagepassing as a fundamental construct with which highly-scalable and highly-reliable distributed systems are built.

Despite the increasing popularity of such programming models, little is known about precise algorithmic reasoning. This is perhaps not without good reason: decision problems such as state-reachability for programs communicating with

* Partially supported by the project ANR-09-SEGI-016 Veridyc.

${ }^{\dagger}$ Supported by a Fondation Sciences Mathématiques de Paris post-doctoral fellowship. 
unbounded reliable queues are undecidable [9], even when there is only a single finite-state process (posting messages to itself). Furthermore, the known decidable under-approximations (e.g., bounding the size of queues) represent queues explicitly, are thus doomed to combinatorial explosion as the size and variability of queue content increases.

Some have proposed analyses which abstract message arrival order [22, 13, 12, or assume messages can be arbitrarily lost 2,3 . Such analyses do not suffice when correctness arguments rely on reliable messaging - several systems specifically do ensure the ordered delivery of messages, including Scala, and recent web-browser specifications [1. Others have proposed analyses which compute finite symbolic representations of queue contents [5, 7]. Known bounded analyses which model queues precisely either bound the maximum capacity of message-queues, ignoring executions which exceed the bound, or bound the total number of process "contexts" 20, 15, where each context involves a single process sending and receiving messages. For each of these bounding schemes there are trivial systems which cannot be adequately explored, e.g., by sending more messages than the allowed queue-capacity, having more processes than contexts, or by alternating message-sends to two processes - we discuss such examples in Section 3. All of the above techniques represent queues explicitly, though perhaps symbolically, and face combinatorial explosion as queue content and variation increase.

In this work we propose a novel technique for bounded analysis of asynchronous message-passing programs with reliable, ordered message queues. Our bounding parameter, introduced in Section 3, is not sensitive to the capacity nor content of message queues, nor the number of process contexts. Instead, we bound the number of process communication cycles by labeling each message with a monotonically-increasing phase number. Each time a message chain visits the same process, the phase number must increase. For a given parameter $k$, we only explore behaviors of up to $k$ phases - though $k$ phases can go a long way. In the leader election distributed protocol 23] for example, each election round occurs in 2 phases: in the first phase each process sends capture messages to the others; in the second phase some processes receive accept messages, and those that find themselves majority-winners broadcast elected messages. In these two phases an unbounded number of messages are sent to an unbounded number of processes across an unbounded number of process contexts!

We demonstrate the true strength of phase-bounding by showing in Sections 4 and 5 that the bounded phase executions of a message-passing program can be concisely encoded as a non-deterministic sequential program, in which messagequeues are not explicitly represented. Our so-called "sequentialization" sheds hope for scalable analyses of message-passing programs. In a small set of simple experiments (Section 4), we demonstrate that our phase-bounded encoding scales far beyond known explicit-queue encodings as queue-content increases, and even remains competitive as queue-content is fixed while the number of phases grows. By reducing to sequential programs, we leverage highly-developed sequential program analysis tools for message-passing programs. 


\section{Asynchronous Message-Passing Programs}

We consider a simple multi-processor programming model in which each processor is equipped with a procedure stack and a queue of pending tasks. Initially all processors are idle. When an idle processor's queue is non-empty, the oldest task in its queue is removed and executed to completion. Each task executes essentially a recursive sequential program, which besides accessing its own processor's global storage, can post tasks to the queues of any processor, including its own. When a task does complete, its processor again becomes idle, chooses the next pending task to execute to completion, and so on. The distinction between queues containing messages and queues containing tasks is mostly aesthetic, but in our task-based treatment queues are only read by idle processors; reading additional messages during a task's execution is prohibited. While in principle many message-passing systems, e.g., in Erlang and Scala, allow reading additional messages at any program point, we have observed that common practice is to read messages only upon completing a task [24.

Though similar to Sen and Viswanathan 22]'s model of asynchronous programs, the model we consider has two important distinctions. First, tasks execute across potentially several processors, rather than only one, each processor having its own global state and pending tasks. Second, the tasks of each processor are executed in exactly the order they are posted. For the case of single-processor programs, Sen and Viswanathan [22]'s model can be seen as an abstraction of the model we consider, since there the task chosen to execute next when a processor is idle is chosen non-deterministically among all pending tasks.

\subsection{Program Syntax}

Let Procs be a set of procedure names, Vals a set of values, Exprs a set of expressions, Pids a set of processor identifiers, and let $T$ be a type. Figure 1 gives the grammar of asynchronous message-passing programs. We intentionally leave the syntax of expressions $e$ unspecified, though we do insist Vals contains true and false, and Exprs contains Vals and the (nullary) choice operator $\star$.

Each program $P$ declares a single global variable $g$ and a procedure sequence, each $p \in$ Procs having a single parameter 1 and top-level statement denoted $s_{p}$; as statements are built inductively by composition with control-flow statements, $s_{p}$ describes the entire body of $p$. The set of program statements $s$ is denoted Stmts. Intuitively, a post $\rho p e$ statement is an asynchronous call to a procedure $p$ with argument $e$ to be executed on the processor identified by $\rho$; a self-post to one's own processor is made by setting $\rho$ to _. A program in which all post statements are self-posts is called a single-processor program, and a program without post statements is called a sequential program.

The programming language we consider is simple, yet very expressive, since the syntax of types and expressions is left free, and we lose no generality by considering only single global and local variables. Appendix A lists several syntactic extensions which we use in the source-to-source translations of the subsequent sections, and which easily reduce to the syntax of our grammar. 


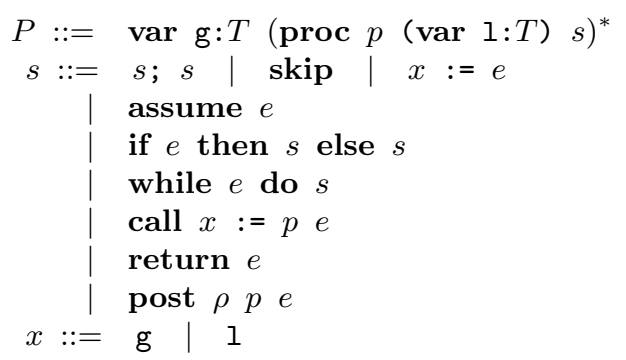

Fig. 1. The grammar of asynchronous message-passing programs $P$. Here $T$ is an unspecified type, and $e, p$, and $\rho$ range, resp., over expressions, procedure names, and processor identifiers.

$$
\begin{gathered}
\frac{\text { Dispatch }}{\langle g, \varepsilon, f \cdot q\rangle \stackrel{\mathrm{S}}{\rightarrow}\langle g, f, q\rangle} \\
\text { Complete } \\
\frac{f=\langle\ell, \text { return } e ; s\rangle}{\langle g, f, q\rangle \stackrel{\mathrm{S}}{\rightarrow}\langle g, \varepsilon, q\rangle} \\
\text { SELF-POST } \\
\frac{s_{1}=\text { post }-p e ; s_{2}}{\ell_{2} \in e\left(g, \ell_{1}\right) \quad f=\left\langle\ell_{2}, s_{p}\right\rangle} \\
\left\langle g,\left\langle\ell_{1}, s_{1}\right\rangle w, q\right\rangle \stackrel{\mathrm{S}}{\rightarrow}\left\langle g,\left\langle\ell_{1}, s_{2}\right\rangle w, q \cdot f\right\rangle
\end{gathered}
$$

Fig. 2. The single-processor transition rules $\rightarrow{ }^{\mathrm{S}}$; see Appendix B for the standard sequential statements.

\subsection{Single-Processor Semantics}

A (procedure) frame $f=\langle\ell, s\rangle$ is a current valuation $\ell \in$ Vals to the procedurelocal variable 1 , along with a statement $s \in$ Stmts to be executed. (Here $s$ describes the entire body of a procedure $p$ that remains to be executed, and is initially set to $p$ 's top-level statement $s_{p}$; we refer to initial procedure frames $t=\left\langle\ell, s_{p}\right\rangle$ as tasks, to distinguish the frames that populate processor queues.) The set of all frames is denoted Frames.

A processor configuration $\kappa=\langle g, w, q\rangle$ is a current valuation $g \in$ Vals to the processor-global variable $\mathrm{g}$, along with a procedure-frame stack $w \in$ Frames* and a pending-tasks queue $q \in$ Frames*. A processor is idle when $w=\varepsilon$. The set of all processor configurations is denoted Pconfigs. A processor configuration map $\xi$ : Pids $\rightarrow$ Pconfigs maps each processor $\rho \in$ Pids to a processor configuration $\xi(\rho)$. We write $\xi(\rho \mapsto \kappa)$ to denote the configuration $\xi$ updated with the mapping $(\rho \mapsto \kappa)$, i.e., the configuration $\xi^{\prime}$ such that $\xi^{\prime}(\rho)=\kappa$, and $\xi^{\prime}\left(\rho^{\prime}\right)=\xi\left(\rho^{\prime}\right)$ for all $\rho^{\prime} \in$ Pids $\backslash\{\rho\}$.

For expressions without program variables, we assume the existence of an evaluation function $\llbracket \cdot \rrbracket_{\mathrm{e}}$ : Exprs $\rightarrow \wp($ Vals $)$ such that $\llbracket \star \rrbracket_{\mathrm{e}}=$ Vals. For convenience we define $e(g, \ell) \stackrel{\text { def }}{=} \llbracket e[g / g, \ell / 1] \rrbracket_{\mathrm{e}}$ to evaluate the expression $e$ in a global valuation $g$ by substituting the current values for variables $g$ and 1 . As these are the only program variables, the substituted expression $e[\mathrm{~g} / \mathrm{g}, \ell / \mathrm{l}]$ has no free variables.

Figure 2 defines the transition relation $\rightarrow^{\mathrm{S}}$ for the asynchronous behavior of each processor; the standard transitions for the sequential statements are listed in Appendix B. The SELf-Post rule creates a new frame to execute the given procedure, and places the new frame in the current processor's pending-tasks queue. The Complete rule returns from the final frame of a task, rendering the processor idle, and the Dispatch rule schedules the least-recently posted task on a idle processor. 


$$
\begin{aligned}
& \frac{\rho_{2} \in \text { enabled }(m, \xi)}{\left\langle\rho_{1}, \xi, m\right\rangle \underset{M}{\longrightarrow}\left\langle\rho_{2}, \xi, m\right\rangle} \\
& \xi_{1}(\rho) \stackrel{\mathrm{S}}{\rightarrow} \kappa \quad \xi_{2}=\xi_{1}(\rho \mapsto \kappa) \\
& \frac{\rho \in \text { enabled }\left(m_{1}, \xi_{1}\right) \quad m_{2}=\operatorname{step}\left(m_{1}, \xi_{1}, \xi_{2}\right)}{\left\langle\rho, \xi_{1}, m_{1}\right\rangle \underset{M}{\longrightarrow}\left\langle\rho, \xi_{2}, m_{2}\right\rangle} \\
& \text { Post } \\
& \xi_{1}\left(\rho_{1}\right)=\left\langle g_{1},\left\langle\ell_{1}, \text { post } \rho_{2} p e ; s\right\rangle w_{1}, q_{1}\right\rangle \\
& \xi_{1}\left(\rho_{2}\right)=\left\langle g_{2}, w_{2}, q_{2}\right\rangle \\
& \rho_{1} \neq \rho_{2} \quad \ell_{2} \in e\left(g_{1}, \ell_{1}\right) \quad f=\left\langle\ell_{2}, s_{p}\right\rangle \\
& \rho_{1} \in \operatorname{enabled}\left(m_{1}, \xi_{1}\right) \quad m_{2}=\operatorname{step}\left(m_{1}, \xi_{1}, \xi_{3}\right) \\
& \xi_{2}=\xi_{1}\left(\rho_{1} \mapsto\left\langle g_{1},\left\langle\ell_{1}, s\right\rangle w_{1}, q_{1}\right\rangle\right) \\
& \xi_{3}=\xi_{2}\left(\rho_{2} \mapsto\left\langle g_{2}, w_{2}, q_{2} \cdot f\right\rangle\right) \\
& \left\langle\rho_{1}, \xi_{1}, m_{1}\right\rangle \underset{M}{\longrightarrow}\left\langle\rho_{1}, \xi_{3}, m_{2}\right\rangle
\end{aligned}
$$

Fig. 3. The multi-processor transition relation $\rightarrow_{M}$ parameterized by a scheduler $M=$ $\langle D$, empty, enabled, step $\rangle$.

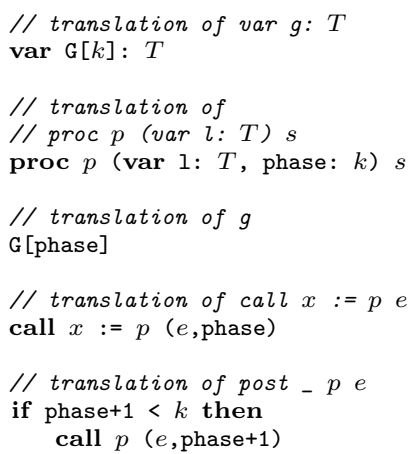

Fig. 4. The $k$-phase sequential translation $((P))_{k}$ of a singleprocessor asynchronous messagepassing program $P$.

\subsection{Multi-Processor Semantics}

In reality the processors of multi-processor systems execute independently in parallel. However, as long as they either do not share memory, or access a sequentially consistent shared memory, it is equivalent, w.r.t. the observations of any single processor, to consider an interleaving semantics: at any moment only one processor executes. In order to later restrict processor interleaving, we make explicit the scheduler which arbitrates the possible interleavings. Formally, a scheduler $M=\langle D$, empty, enabled, step $\rangle$ consists of a data type $D$ of scheduler objects $m \in D$, a scheduler constructor empty $\in D$, a scheduler decision function enabled $:(D \times$ (Pids $\rightarrow$ Pconfigs $)) \rightarrow \wp$ (Pids), and a scheduler update function step $:(D \times$ (Pids $\rightarrow$ Pconfigs $) \times$ (Pids $\rightarrow$ Pconfigs $)) \rightarrow D$. The arguments to enabled allow a scheduler to decide which processors are enabled depending on the execution history. A scheduler is deterministic when $|\operatorname{enabled}(m, \xi)| \leq 1$ for all $m \in D$ and $\xi$ :Pids $\rightarrow$ Pconfigs, and is non-blocking when for all $m$ and $\xi$, if there is some $\rho \in \operatorname{Pids}$ such that $\xi(\rho)$ is either non-idle or has pending tasks, then there exists $\rho^{\prime} \in \operatorname{Pids}$ such that $\rho^{\prime} \in \operatorname{enabled}(m, \xi)$ and $\xi\left(\rho^{\prime}\right)$ is either non-idle or has pending tasks. A configuration $c=\langle\rho, \xi, m\rangle$ is a currently executing processor $\rho \in$ Pids, along with a processor configuration map $\xi$, and a scheduler object $m$.

Figure 3 defines the multi-processor transition relation $\rightarrow_{M}$, parameterized by a scheduler $M$. The SwITCH rule non-deterministically schedules any enabled processor, while the STEP rule executes one single-processor program step on the currently scheduled processor, and updates the scheduler object. Finally, the PosT rule creates a new frame to execute the given procedure, and places the the new frame on the target processor's pending-tasks queue. 
Until further notice, we assume $M$ is a completely non-deterministic scheduler; i.e., all processors are always enabled. In Section 5 we discuss alternatives.

An $M$-execution of a program $P$ (from $c_{0}$ to $c_{j}$ ) is a configuration sequence $c_{0} c_{1} \ldots c_{j}$ such that $c_{i} \rightarrow_{M} c_{i+1}$ for $0 \leq i<j$. An initial condition $\iota=\left\langle\rho_{0}, g_{0}, \ell_{0}, p_{0}\right\rangle$ is a processor identifier $\rho_{0}$, along with a global-variable valuation $g_{0} \in$ Vals, a local-variable valuation $\ell_{0} \in$ Vals, and a procedure $p_{0} \in$ Procs. A configuration $c=\left\langle\rho_{0}, \xi\right.$, empty $\rangle$ of a program $P$ is $\left\langle\rho_{0}, g_{0}, \ell_{0}, p_{0}\right\rangle$-initial when $\xi\left(\rho_{0}\right)=\left\langle g_{0}, \varepsilon,\left\langle\ell_{0}, s_{p_{0}}\right\rangle\right\rangle$ and $\xi(\rho)=\left\langle g_{0}, \varepsilon, \varepsilon\right\rangle$ for all $\rho \neq \rho_{0}$. A configuration $\langle\rho, \xi, m\rangle$ is $g_{f}$-final when $\xi\left(\rho^{\prime}\right)=\left\langle g_{f}, w, q\right\rangle$ for some $\rho^{\prime} \in$ Pids, and $w, q \in$ Frames*. We say a global valuation $g$ is $M$-reachable in $P$ from $\iota$ when there exists an $M$-execution of $P$ from some $c_{0}$ to some $c$ such that $c_{0}$ is $\iota$-initial and $c$ is $g$-finat

Definition 1. The state-reachability problem is to determine for an initial condition $\iota$, valuation $g$, and program $P$, whether $g$ is reachable in $P$ from $\iota$.

\section{Phase-Bounded Execution}

Because processors execute tasks precisely in the order which they are posted to their unbounded task-queues, our state-reachability problem is undecidable, even with only a single processor accessing finite-state data [9]. Since it is not algorithmically possible to consider every execution precisely, in what follows we present an incremental under-approximation. For a given bounding parameter $k$, we consider a subset of execution (prefixes) precisely; as $k$ increases, the set of considered executions increases, and in the limit as $k$ approaches infinity, every execution of any program is considered - though for many programs, every execution is considered with a finite value of $k$.

In a given execution, a task-chain $t_{1} t_{2} \ldots t_{i}$ from $t_{1}$ to $t_{i}$ is a sequence of task $2^{2}$ such that the execution of each $t_{j}$ posts $t_{j+1}$, for $0<j<i$, and we say that $t_{1}$ is an ancestor of $t_{i}$. We characterize execution prefixes by labeling each task $t$ posted in an execution with a phase number $\varphi(t) \in \mathbb{N}$ :

$$
\varphi(t)= \begin{cases}0 & \text { if } t \text { is initially pending. } \\ \varphi\left(t^{\prime}\right) & \text { if } t \text { is posted to processor } \rho \text { by } t^{\prime} \\ \varphi\left(t^{\prime}\right)+1 & \text { and } t \text { has no phase- } \varphi\left(t^{\prime}\right) \text { ancestor on processor } \rho .\end{cases}
$$

For instance, considering Figure 5 a, supposing all on a single processor, an initial task $A_{1}$ posts $A_{2}, A_{3}$, and $A_{4}$, then $A_{2}$ posts $A_{5}$ and $A_{6}$, and then $A_{3}$ posts $A_{7}$, which in turn posts $A_{8}$ and $A_{9}$. Task $A_{1}$ has phase 0 . Since each post is made to the same processor, the phase number is incremented for each posted task. Thus the phase 1 tasks are $\left\{A_{2}, A_{3}, A_{4}\right\}$, the phase 2 tasks are $\left\{A_{5}, A_{6}, A_{7}\right\}$, and the phase 3 tasks are $\left\{A_{8}, A_{9}\right\}$. Notice that tasks of a given phase only

\footnotetext{
${ }^{1}$ In the presence of the assume statement, only the values reached in completed executions are guaranteed to be valid.

${ }^{2}$ We assume each task in a given execution has implicitly a unique task-identifier.
} 


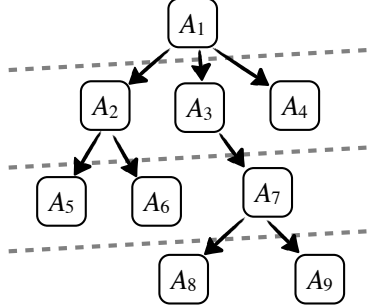

(a)

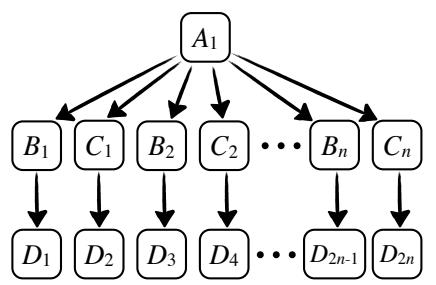

(b)

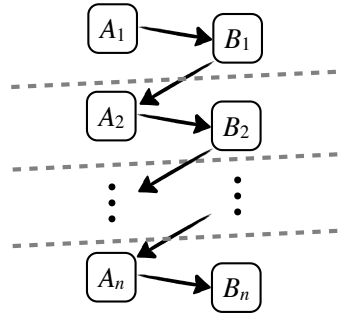

(c)

Fig. 5. Phase-bounded executions with processors $A, B, C$, and $D$; each task's label (e.g., $\left.A_{i}\right)$ indicates the processor it executes on (e.g., $A$ ). Arrows indicate the posting relation, indices indicate execution order on a given processor, and dotted lines indicate phase boundaries.

execute after all tasks of the previous phase have completed, i.e., execution order is in phase order; only executing tasks up to a given phase does correspond to a valid execution prefix.

Definition 2. An execution is $k$-phase when $\varphi(t)<k$ for each executed task $t$.

The execution in Figure $5 \mathrm{a}$ is a 4 -phase execution, since all tasks have phase less than 4 . Despite there being an arbitrary number $4 n+1$ of posted tasks, the execution in Figure $5 \mathrm{~b}$ is 1-phase, since there are no task-chains between same-processor tasks. Contrarily, the execution in Figure 5c requires $n$ phases to execute all $2 n$ tasks, since every other occurrence of an $A_{i}$ task creates a task-chain between $A$-tasks.

Note that bounding the number of execution phases does not necessarily bound the total number of tasks executed, nor the maximum size of task queues, nor the amount of switching between processors. Instead, a bound $k$ restricts the maximum length of task chains to $k \cdot \mid$ Pids $\mid$. In fact, phase-bounding is incomparable to bounding the maximum size of task queues. On the one hand, every execution of a program in which one root task posts an arbitrary, unbounded number of tasks to other processors (e.g., in Figure 5b) are explored with 1 phase, though no bound on the size of queues will capture all executions. On the other hand, all executions with a single arbitrarily-long chain of tasks (e.g., in Figure 55) are explored with size 1 task queues, though no limited number of phases captures all executions. In the limit as the bounding parameter increases, either scheme does capture all executions.

Theorem 1 (Completeness). For every execution $h$ of a program $P$, there exists $k \in \mathbb{N}$ such that $h$ is a $k$-phase execution.

\section{Phase-Bounding for Single-Processor Programs}

Characterizing executions by their phase-bound reveals a simple and efficient technique for bounded exploration. This seems remarkable, given that phase- 
bounding explores executions in which arbitrarily many tasks execute, making the task queue arbitrarily large. The first key ingredient is that once the number of phases is bounded, each phase can be executed in isolation. For instance, consider again the execution of Figure 5 a. In phase 1 , the tasks $A_{2}, A_{3}$, and $A_{4}$ pick up execution from the global valuation $g_{1}$ which $A_{1}$ left off at, and leave behind a global valuation $g_{2}$ for the phase 2 tasks. In fact, given the sequence of tasks in each phase, the only other "communication" between phases is a single passed global valuation; executing that sequence of tasks on that global valuation is a faithful simulation of that phase.

The second key ingredient is that the ordered sequence of tasks executed in a given phase is exactly the ordered sequence of tasks posted in the previous phase. This is obvious, since tasks are executed in the order they are posted. However, combined with the first ingredient we have quite a powerful recipe. Supposing the global state $g_{i}$ at the beginning of each phase $i$ is known initially, we can simulate a $k$-phase execution by executing each task posted to phase $i$ as soon as it is posted, with an independent virtual copy of the global state, initially set to $g_{i}$. That is, our simulation will store a vector of $k$ global valuations, one for each phase. Initially, the $i^{\text {th }}$ global valuation is set to the state $g_{i}$ in which phase $i$ begins; tasks of phase $i$ then read from and write to the $i^{\text {th }}$ global valuation. It then only remains to ensure that the global valuations $g_{i}$ used at the beginning of each phase $0<i<k$ match the valuations reached at the end of phase $i-1$.

This simulation is easily encoded into a non-deterministic sequential program with $k$ copies of global storage. The program begins by non-deterministically setting each copy to an arbitrary value. Each task maintains their current phase number $i$, and accesses the $i^{\text {th }}$ copy of global storage. Each posted task is simply called instead of posted, its phase number set to one greater than its parentposts to tasks with phase number $k$ are ignored. At the end of execution, the program ensures that the $i^{\text {th }}$ global valuation matches the initially-used valuation for phase $i+1$, for $0 \leq i<k-1$. When this condition holds, any global valuation observed along the execution is reachable within $k$ phases in the original program. Figure 4 lists a code-to-code translation which implements this simulation.

Theorem 2. A global-valuation $g$ is reachable in a $k$-phase execution of a singleprocessor program $P$ if and only if $g$ is reachable in $((P))_{k}$ - the $k$-phase sequential translation of $P$.

When the underlying sequential program model has a decidable state-reachability problem, Theorem 2 gives a decision procedure for the phase-bounded statereachability problem, by applying the decision procedure for the underlying model to the translated program. This allows us for instance to derive a decidability result for programs with finite data domains.

Corollary 1. The k-phase state-reachability problem is decidable for singleprocessor programs with finite data domains.

More generally, given any underlying sequential program model, our translation makes applicable any analysis tool for said model to message-passing 
programs, since the values of the additional variables are either from the finite domain $\{0, \ldots, k-1\}$, or in the domain of the original program variables.

Note that our simulation of a $k$-phase execution does not explicitly store the unbounded task queue. Instead of storing a multitude of possible unbounded task sequences, our simulation stores exactly $k$ global state valuations. Accordingly, our simulation is not doomed to the unavoidable combinatorial explosion encountered by storing (even bounded-size) task queues explicitly. To demonstrate the capability of our advantage, we measure the time to verify two fabricated yet illustrative examples (listed in full in Appendix C, comparing our bounded-phase encoding with a bounded task-queue encoding. In the bounded task-queue encoding, we represent the task-queue explicitly by an array of integers, which stores the identifiers of posted procedures 3 . When control of the initial task completes, the program enters a loop which takes a procedure identifier from the head of the queue, and calls the associated procedure. When the queue reaches a given bound, any further posted tasks are ignored.

The first program $P_{1}(i)$, parameterized by $i \in \mathbb{N}$, has a single Boolean global variable $\mathrm{b}, i$ procedures named $p_{1}, \ldots, p_{i}$, which assert $\mathrm{b}$ to be false and set $\mathrm{b}$ to true, and $i$ procedures named $q_{1}, \ldots, q_{i}$ which set $\mathrm{b}$ to false. Initially, $P_{1}(i)$ sets $\mathrm{b}$ to false, and enters a loop in which each iteration posts some $p_{j}$ followed by some $q_{j}$. Since a $q_{j}$ task must be executed between each $p_{j}$ task, each of the assertions are guaranteed to hold. Figure 6 a compares the time required to verify $P_{1}(i)$ (using the BoogIE verification engine 4]) for various values of $i$, and various bounds $n$ on loop unrolling. Note that although every execution of $P_{1}(i)$ has only 2 phases, to explore all $n$ loop iterations in any given execution, the size of queues must be at least $2 n$, since two tasks are posted per iteration. Even for this very simple program, representing (even bounded) task-queues explicitly does not scale, since the number of possible task-queues grows astronomically as the size of task-queues grow. This ultimately prohibits the bounded tasks-queue encodings from exploring executions in which more than a mere few simple tasks execute. On the contrary, our bounded-phase simulation easily explores every execution up to the loop-unrolling bound in a few seconds.

To be fair, our second program $P_{2}$ is biased to support the bounded task-queue encoding. Following the example of Figure 5c, $P_{2}$ again has a single Boolean global variable $\mathrm{b}$, and two procedures: $p_{1}$ asserts $\mathrm{b}$ to be false, sets $\mathrm{b}$ to true, and posts $p_{2}$, while $p_{2}$ sets $\mathrm{b}$ to false and posts $p_{1}$. Initially, the program $P_{2}$ sets $\mathrm{b}$ to false and posts a single $p_{1}$ task. Again here, since a $p_{2}$ task must execute between each $p_{1}$ task, each of the assertions are guaranteed to hold. Figure $6 \mathrm{~b}$ compares the time required to verify $P_{2}$ for various bounds $n$ on the number of tasks explored 4 . Note that although every execution of $P_{2}$ uses only size 1 task-queues, to explore all $n$ tasks in any given execution, the number of phases

\footnotetext{
${ }^{3}$ For simplicity our examples do not pass arguments to tasks; in general, one should also store in the task-queue array the values of arguments passed to each posted procedure.

${ }^{4}$ The number $n$ of explored tasks is controlled by limiting the number of loop unrollings in the bounded task-queue encoding, and limiting the recursion depth, and phase-bound, in the bounded-phase encoding.
} 


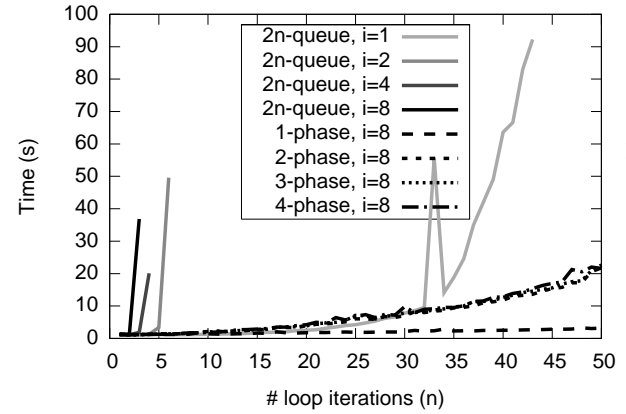

(a)

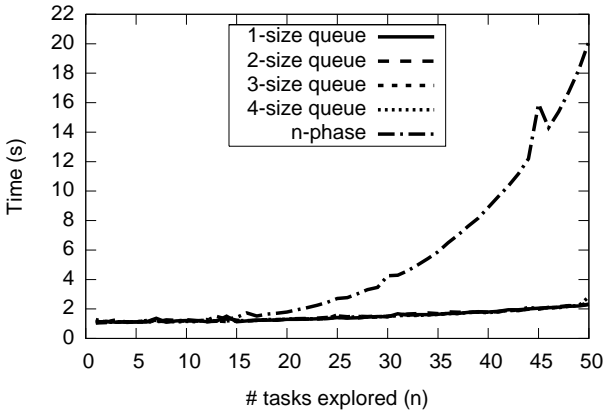

(b)

Fig. 6. Time required to verify (a) the program $P_{1}(i)$, and (b) the program $P_{2}$ with the BoOGIE verification engine using various encodings (bounded queues, bounded phase), and various loop unrolling bounds. Time-out is set to $100 \mathrm{~s}$.

must be at least $n$, since each task must execute in its own phase. Although verification time for the bounded-phase encoding does increase with $n$ faster than the bounded task-queue encoding - as expected - due to additional copies of the global valuation, and more deeply in-lined procedures, the verification time remains manageable. In particular, the time does not explode uncontrollably: even 50 tasks are explored in under 20 s.

\section{Phase-Bounding for Multi-Processor Programs}

Though state-reachability under a phase bound is immediately and succinctly reducible to sequential program analysis for single-processor programs, the multiprocessor case is more complicated. The added complexity arises due to the many orders in which tasks on separate processors can contribute to others' task-queues. As a simple example, consider the possible bounded-phase executions of Figure $5 \mathrm{~b}$ with four processors, $A, B, C$, and $D$. Though $B$ 's tasks $B_{1}, \ldots, B_{n}$ must be executed in order, and $C$ 's tasks $C_{1}, \ldots, C_{n}$ must also be executed in order, the order of $D$ 's tasks are not pre-determined: the arrival order of $D$ 's tasks depends on how $B$ 's and $C$ 's tasks interleave. Suppose for instance $B_{1}$ executes to completion before $C_{1}$, which executes to completion before $B_{2}$, and so on. In this case $D$ 's tasks arrive to $D$ 's queue, and ultimately execute, in the index order $D_{1}, D_{2}, \ldots$ as depicted. However, there exist executions for every possible order of $D$ 's tasks respecting $D_{1}<D_{3}<\ldots$ and $D_{2}<D_{4}<\ldots$ (where $<$ denotes an ordering constraint) - many possible orders indeed! In fact, due to the capability of such unbounded interleaving, the problem of state-reachability under a phase-bound is undecidable for multi-processor programs, even for programs with finite data domains.

Theorem 3. The k-phase bounded state-reachability problem is undecidable for multi-processor programs with finite data domains. 
Note that Theorem 3 holds independently of whether memory is shared between processors: the fact that a task-queue can store any possible (unbounded) shuffling of tasks posted by two processors lends the power to simulate Post's correspondence problem [19].

Theorem 3 insists that phase-bounding alone will not lead to the elegant encoding to sequential programs which was possible for single-processor programs. If that were possible, then the translation from a finite-data program would lead to a finite-data sequential program, and thus a decidable state-reachability problem. Since a precise algorithmic solution to bounded-phase state-reachability is impossible for multi-processor programs, we resort to a further incremental yet orthogonal under-approximation, which limits the number of considered processor interleavings. The following development is based on delay-bounded scheduling [11].

We define a delaying scheduler $M=\langle D$, empty, enabled, step, delay $\rangle$, as a scheduler $\langle D$, empty, enabled, step $\rangle$, along with a function delay : $(D \times$ Pids $\times$ (Pids $\rightarrow$ Pconfigs) $) \rightarrow D$. Furthermore, we extend the transition relation of Figure 3 with a postponing rule of Figure 7 which we henceforth refer to as a delay (operation), saying that processor $\rho$ is delayed. Note that a delay operation may or may not change the set of enabled processors in any given step, depending on the scheduler. A delaying scheduler is delay-accessible when for every configuration $c_{1}$ and non-idle or task-pending processor $\rho$, there exists a sequence $c_{1} \rightarrow_{M} \ldots \rightarrow_{M}$ $c_{j}$ of DeLAY-steps such that $\rho$ is enabled in $c_{j}$. Given executions $h_{1}$ and $h_{2}$ of (delaying) schedulers $M_{1}$ and $M_{2}$ resp., we write $h_{1} \sim h_{2}$ when $h_{1}$ and $h_{2}$ are identical after projecting away delay operations.

Definition 3. An execution with at most $k$ delay operators is called $k$-delay.

Consider again the possible executions of Figure $5 \mathrm{p}$, but suppose we fix a deterministic scheduler $M$ which without delaying would execute $D$ 's tasks in index order: $D_{1}, D_{2}, \ldots$; furthermore suppose that delaying a processor $\rho$ in phase $i$ causes $M$ to execute the remaining phase $i$ tasks of $\rho$ in phase $i+1$, while keeping the tasks of other processors in their current phase. Without using any delays, the execution of Figure $5 \mathrm{~b}$ is unique, since $M$ is deterministic. However, as Figure 8 illustrates, using a single delay, it is possible to also derive the order $D_{1}, D_{3}, \ldots, D_{2 n-1}, D_{2}, D_{4}, \ldots, D_{2 n}$ (among others): simply delay processor $C$ once before $C_{1}$ posts $D_{2}$. Since this forces the $D_{2 i}$ tasks posted by each $C_{i}$ to occur in the second phase, it follows they must all happen after the $D_{2 i-1}$ tasks posted by each $B_{i}$.

Theorem 4 (Completeness). Let $M$ be any delay-accessible scheduler. For every execution $h$ of a program $P$, there exists an $M$-execution $h^{\prime}$ and $k \in \mathbb{N}$ such that $h^{\prime}$ is a $k$-delay execution and $h^{\prime} \sim h$.

Note that Theorem 4 holds for any delay-accessible scheduler $M$-even deterministic schedulers. As it turns out there is one particular scheduler $M_{\mathrm{bfs}}$ for which we know a convenient sequential encoding, and this scheduler is described in Appendix $\mathrm{D}$. For the moment, the important points to note are that $M_{\mathrm{bfs}}$ 
$\frac{m_{2}=\operatorname{delay}\left(m_{1}, \rho, \xi\right)}{\left\langle\rho, \xi, m_{1}\right\rangle \underset{M}{\longrightarrow}\left\langle\rho, \xi, m_{2}\right\rangle}$

Fig. 7. The delay operation.

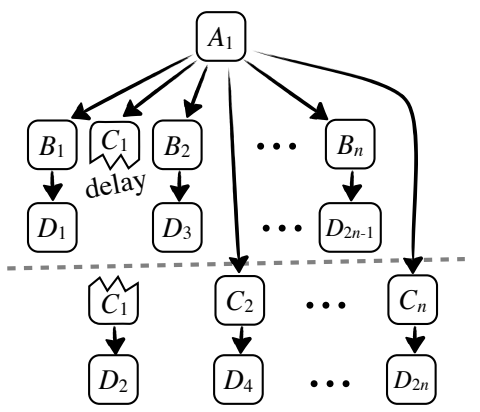

Fig. 8. A 2-phase delaying execution varying the 1-phase execution of Figure 5 p.

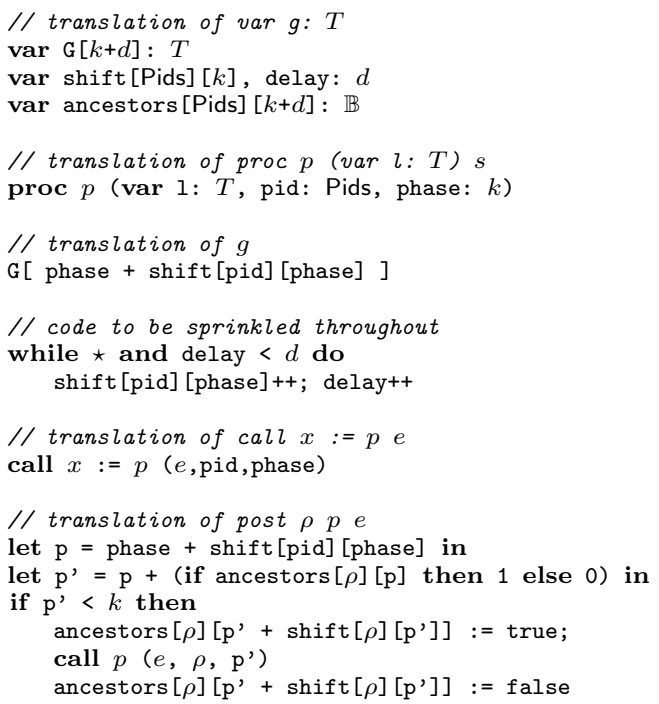

Fig. 9. The $k$-phase $d$-delay sequential translation $((P))_{k, d}^{\mathrm{bfs}}$ of a multi-processor message-passing asynchronous program $P$.

is deterministic, non-blocking, and delay-accessible. Essentially, determinism allows us to encode the scheduler succinctly in a sequential program; the nonblocking property ensures this scheduler does explore some execution, rather than needlessly ceasing to continue; delay-accessibility combined with Theorem 4 ensure the scheduler is complete in the limit. Figure 9 lists a code-to-code translation which encodes bounded-phase and bounded-delay exploration of a given program according to the $M_{\mathrm{bfs}}$ scheduler as a sequential program.

Our translation closely follows the single-processor translation of Section 4 the key differences being:

- the phase of a posted task is not necessarily incremented, since posted tasks may not have same-processor ancestors in the current phase, and

- at any point, the currently executing task may increment a delay counter, causing all following tasks on the same processor to shift forward one additional phase.

As the global values reached by each processor at the end of each phase $i-1$ must be ensured to match the initial values of phase $i$, for $0<i<k+d$, so must the values for the shift counter: an execution is only valid when for each processor $\rho \in$ Pids and each phase $0<i<k$, shift $[\rho][i-1]$ matches the initial value of shift $[\rho][i]$.

Theorem 5. A global valuation $g$ is reachable in a $k$-phase d-delay $M_{\mathrm{bfs}}$-execution of a multi-processor program $P$ if and only if $g$ is reachable in $((P))_{k, d}^{b f s}$. 
As is the case for our single-processor translation, our simulation does not explicitly store the unbounded tasks queue, and is not doomed to combinatorial explosion faced by storing tasks-queues explicitly.

\section{Related Work}

Our work follows the line of research on compositional reductions from concurrent to sequential programs. The initial so-called "sequentialization" 21] explored multi-threaded programs up to one context-switch between threads, and was later expanded to handle a parameterized amount of context-switches between a statically-determined set of threads executing in round-robin order [20, 17. La Torre et al. [16] later extended the approach to handle programs parameterized by an unbounded number of statically-determined threads, and shortly after, Emmi et al. 11] further extended these results to handle an unbounded amount of dynamically-created tasks, which besides applying to multi-threaded programs, naturally handles asynchronous event-driven programs 22]. Bouajjani et al. 8] pushed these results even further to a sequentialization which attempts to explore as many behaviors as possible within a given analysis budget. Each of these sequentializations necessarily do provide a bounding parameter which limits the amount of interleaving between threads or tasks, but none are capable of precisely exploring tasks in creation order, which is abstracted away from their program models [22. Kidd et al. [14]'s sequentialization is sensitive to task priorities, their reduction assumes a finite number of statically-determined tasks.

In a closely-related work, La Torre et al. [15 propose a "context-bounded" analysis of shared-memory multi-pushdown systems communicating with messagequeues. According to this approach, one "context" involves a single process reading from its queue, and posting to the queues of other processes, and the number of contexts per execution is bounded. Our work can be seen as an extension in a few ways. First, and most trivially, in their setting a process cannot post to its own message queue; this implies that at least $2 k$ contexts must be used to simulate $k$ phases of a single-processor program. Second, there are families of 1-phase executions which require an unbounded number of task-contexts to capture; the execution order $D_{1} D_{2} D_{3} \ldots D_{2 n}$ of Figure $5 \mathrm{~b}$ is such an example. We conjecture that bounded phase and delay captures context-bounding - i.e., there exists a polynomial function $f: \mathbb{N} \rightarrow \mathbb{N}$ such that every $k$-context bounded execution of any program $P$ is also a $f(k)$-phase and delay bounded execution. Finally, though phase-bounding leads to a convenient sequential encoding, we are unaware whether a similar encoding is possible for context-bounding.

Boigelot and Godefroid [5] and Bouajjani and Habermehl [ [ analyses of message-passing programs by computing explicit finite symbolic representations of message-queues. As our sequentialization does not represent queues explicitly, we do not restrict the content of queues to convenientlyrepresentable descriptions. Furthermore, reduction to sequential program analyses is easily implementable, and allows us to leverage highly-developed and optimized program analysis tools. 


\section{Conclusion}

By introducing a novel phase-based characterization of message-passing program executions, we enable bounded program exploration which is not limited by message-queue capacity nor the number of processors. We show that the resulting phase-bounded analysis problems can be solved by concise reduction to sequential program analysis. Preliminary evidence suggests our approach is at worst competitive with known task-order respecting bounded analysis techniques, and can easily scale where those techniques quickly explode.

\section{Acknowledgments}

We thank Constantin Enea, Cezara Dragoi, Pierre Ganty, and the anonymous reviewers for helpful feedback.

\section{References}

[1] HTML5: A vocabulary and associated APIs for HTML and XHTML. http: //dev.w3.org/htm15/spec/Overview.html.

[2] P. A. Abdulla and B. Jonsson. Verifying programs with unreliable channels. In LICS '93: Proc. 8th Annual IEEE Symposium on Logic in Computer Science, pages 160-170. IEEE Computer Society, 1993.

[3] P. A. Abdulla, A. Bouajjani, and B. Jonsson. On-the-fly analysis of systems with unbounded, lossy fifo channels. In $C A V$ '98: Proc. 10th International Conference on Computer Aided Verification, volume 1427 of LNCS, pages 305-318. Springer, 1998.

[4] M. Barnett, K. R. M. Leino, M. Moskal, and W. Schulte. Boogie: An intermediate verification language. http://research.microsoft.com/en-us/ projects/boogie/.

[5] B. Boigelot and P. Godefroid. Symbolic verification of communication protocols with infinite state spaces using QDDs. Formal Methods in System Design, 14(3):237-255, 1999.

[6] A. Bouajjani and P. Habermehl. Symbolic reachability analysis of fifochannel systems with nonregular sets of configurations. Theor. Comput. Sci., 221(1-2):211-250, 1999.

[7] A. Bouajjani, P. Habermehl, and T. Vojnar. Verification of parametric concurrent systems with prioritised FIFO resource management. Formal Methods in System Design, 32(2):129-172, 2008.

[8] A. Bouajjani, M. Emmi, and G. Parlato. On sequentializing concurrent programs. In SAS '11: Proc. 18th International Symposium on Static Analysis, volume 6887 of LNCS, pages 129-145. Springer, 2011.

[9] D. Brand and P. Zafiropulo. On communicating finite-state machines. J. ACM, 30(2):323-342, 1983.

[10] R. Dahl. Node.js: Evented I/O for V8 JavaScript. http://nodejs.org/. 
[11] M. Emmi, S. Qadeer, and Z. Rakamarić. Delay-bounded scheduling. In POPL '11: Proc. 38th ACM SIGPLAN-SIGACT Symposium on Principles of Programming Languages, pages 411-422. ACM, 2011.

[12] P. Ganty and R. Majumdar. Algorithmic verification of asynchronous programs. CoRR, abs/1011.0551, 2010. http://arxiv.org/abs/1011.0551.

[13] R. Jhala and R. Majumdar. Interprocedural analysis of asynchronous programs. In POPL '07: Proc. 34th ACM SIGPLAN-SIGACT Symposium on Principles of Programming Languages, pages 339-350. ACM, 2007.

[14] N. Kidd, S. Jagannathan, and J. Vitek. One stack to run them all: Reducing concurrent analysis to sequential analysis under priority scheduling. In SPIN '10: Proc. 17th International Workshop on Model Checking Software, volume 6349 of $L N C S$, pages 245-261. Springer, 2010.

[15] S. La Torre, P. Madhusudan, and G. Parlato. Context-bounded analysis of concurrent queue systems. In TACAS '08: Proc. 14th International Conference on Tools and Algorithms for the Construction and Analysis of Systems, volume 4963 of LNCS, pages 299-314. Springer, 2008.

[16] S. La Torre, P. Madhusudan, and G. Parlato. Model-checking parameterized concurrent programs using linear interfaces. In $C A V$ '10: Proc. 22nd International Conference on Computer Aided Verification, volume 6174 of LNCS, pages 629-644. Springer, 2010.

[17] A. Lal and T. W. Reps. Reducing concurrent analysis under a context bound to sequential analysis. Formal Methods in System Design, 35(1):73-97, 2009.

[18] M. S. Miller, E. D. Tribble, and J. S. Shapiro. Concurrency among strangers. In TGC '05: Proc. International Symposium on Trustworthy Global Computing, volume 3705 of $L N C S$, pages 195-229. Springer, 2005.

[19] E. L. Post. A variant of a recursively unsolvable problem. Bull. Amer. Math. Soc, 52(4):264-268, 1946.

[20] S. Qadeer and J. Rehof. Context-bounded model checking of concurrent software. In TACAS '05: Proc. 11th International Conference on Tools and Algorithms for the Construction and Analysis of Systems, volume 3440 of LNCS, pages 93-107. Springer, 2005.

[21] S. Qadeer and D. Wu. KISS: Keep it simple and sequential. In PLDI '04: Proc. ACM SIGPLAN Conference on Programming Language Design and Implementation, pages 14-24. ACM, 2004.

[22] K. Sen and M. Viswanathan. Model checking multithreaded programs with asynchronous atomic methods. In $C A V$ '06: Proc. 18th International Conference on Computer Aided Verification, volume 4144 of LNCS, pages 300-314. Springer, 2006.

[23] H. Svensson and T. Arts. A new leader election implementation. In Erlang '05: Proc. ACM SIGPLAN Workshop on Erlang, pages 35-39. ACM, 2005.

[24] F. Trottier-Hebert. Learn you some Erlang for great good! http: //learnyousomeerlang.com/ 


\section{A Syntactic Extensions Used in Our Code Translations}

The following syntactic extensions are reducible to the original program syntax of Section 2.1. Here we freely assume the existence of various type- and expressionconstructors. This does not present a problem since our program semantics does not restrict the language of types nor expressions.

Multiple types. Multiple type labels $T_{1}, \ldots, T_{j}$ can be encoded by systematically replacing each $T_{i}$ with the sum-type $T=\sum_{i=1}^{j} T_{i}$. This allows local and global variables with distinct types.

Multiple variables. Additional variables $\mathrm{x}_{1}: T_{1}, \ldots, \mathrm{x}_{j}: T_{j}$ can be encoded with a single record-typed variable $\mathrm{x}: T$, where $T$ is the record type

$$
\left\{\mathrm{f}_{1}: T_{1}, \ldots, \mathrm{f}_{j}: T_{j}\right\}
$$

and all occurrences of $\mathrm{x}_{i}$ are replaced by $\mathrm{x} . \mathrm{f}_{i}$. When combined with the extension allowing multiple types, this allows each procedure to declare any number and type of local variable parameters, distinct from the number and type of global variables.

Local variable declarations. Additional (non-parameter) local variable declarations var l': $T$ to a procedure $p$ can be encoded by adding l' to the list of parameters, and systematically adding an initialization expression (e.g., the choice expression

$\star$, or false) to the corresponding position in the list of arguments at each call site of $p$ to ensure that $I$ ' begins correctly (un)initialized.

Unused values. Call assignments call $\mathrm{x}:=p e$, where $\mathrm{x}$ is not subsequently used, can be written as call _ $:=p e$, where _ $: T$ is an additional unread local variable, or simpler yet as call $p e$.

Unused branches. if $e$ then $s$ else skip is abbreviated by if $e$ then $s$.

Increment. Increment operations $x++$ are encoded as $x:=x+1$.

Let bindings. Let bindings of the form let $\mathrm{x}: T=e$ in can be encoded by declaring $\mathrm{x}$ as a local variable var $\mathrm{x}$ : $T$ immediately followed by an assignment $\mathrm{x}:=e$. This construct is used to explicate that the value of $\mathrm{x}$ remains constant once initialized. The binding let $\mathrm{x}: T$ in is encoded by the binding let $\mathrm{x}: T=\star$ in where $\star$ is the choice expression.

Arrays. Finite arrays with $j$ elements of type $T$ can be encoded as records of type $\left\{f_{1}: T, \ldots, f_{j}: T\right\}$, where $f_{1} \ldots f_{j}$ are fresh names. Occurrences of terms a [i] are replaced by a. $f_{i}$, and array-expressions $\left[e_{1}, \ldots, e_{j}\right]$ are replaced by record-expressions $\left\{\mathrm{f}_{1}=e_{1}, \ldots, \mathrm{f}_{j}=e_{j}\right\}$. 


\section{B Sequential Program Semantics}

For expressions without program variables, we assume the existence of an evaluation function $\llbracket \cdot \rrbracket_{\mathrm{e}}:$ Exprs $\rightarrow \wp($ Vals $)$ such that $\llbracket \star \rrbracket_{\mathrm{e}}=$ Vals. For convenience, given a processor configuration $\kappa=\langle g, w, q\rangle$ and $w=\langle\ell, s\rangle w^{\prime}$, we define

$$
e(\kappa) \stackrel{\text { def }}{=} e(g, \ell) \stackrel{\text { def }}{=} \llbracket e[g / g, \ell / 1] \rrbracket_{\mathrm{e}}
$$

to evaluate the expression $e$ in a processor configuration $\kappa$ (alternatively, in a global valuation $g$ and local valuation $\ell$ ) by substituting the current values for variables $g$ and 1 . As these are the only program variables, the substituted expression $e[g / \mathrm{g}, \ell / \mathrm{l}]$ has no free variables. Additionally we define

$$
\begin{array}{rr}
\kappa\left(\mathrm{g} \leftarrow g^{\prime}\right) \stackrel{\text { def }}{=}\left\langle g^{\prime}, w, q\right\rangle & \text { global assignment, } \\
\kappa\left(1 \leftarrow \ell^{\prime}\right) \stackrel{\text { def }}{=}\left\langle g,\left\langle\ell^{\prime}, s\right\rangle w^{\prime}, q\right\rangle & \text { local assignment, } \\
\kappa \cdot f \stackrel{\text { def }}{=}\langle g, f \cdot w, q\rangle & \text { append stack frame. }
\end{array}
$$

To further reduce clutter in the operational program semantics, we introduce a notion of context. A statement context $S$ is a term derived from the grammar $S::=\diamond \mid S$; $s$, where $s \in$ Stmts. We write $S[s]$ for the statement obtained by substituting a statement $s$ for the unique occurrence of $\diamond$ in $S$. Intuitively, a context filled with $s$, e.g., $S[s]$, indicates that $s$ is the next statement to execute in the statement sequence $S[s]$. Similarly, a processor configuration context $C=\langle g,\langle\ell, S\rangle w, q\rangle$ is a processor configuration whose top-most frame's statement is replaced with a statement context, and we write $C[s]$ to denote the processor configuration $\langle g,\langle\ell, S[s]\rangle w, q\rangle$. When $e$ is an expression, we abbreviate $e(C[$ skip $])$ by $e(C)$.

Figure 10 defines the transition relation $\rightarrow^{\mathrm{S}}$ for the standard sequential program statements. The SkIP rule simply steps past the skip statement. The Assume rule proceeds only when the given expression $e$ evaluates to true. The Assign statement stores the value of a given expression in either the local variable 1 or the global variable $g$. The IF-THEN and IF-ELSE rules proceeds to either the then or else branch, depending on the current valuation of the given expression $e$. Similarly, the Loop-Do and Loop-End rules proceed to (re-)enter the loop when the given expression $e$ evaluates to true, and step past the loop when $e$ evaluates to false. More interestingly, the CALL rule creates a new procedure frame $f$ by evaluating the given argument $e$, and places $f$ at the top of the procedure-frame stack. The RETURN rule removes the top-most procedure frame from the stack, and substitutes the valuation of the return expression $e$ into the assignment $x:=\star$ left below by the matching call statement. Note that the transition relation $\rightarrow$ is non-deterministic, since the evaluation of an expression $e$ can result in an arbitrary set of possible values.

\section{Full Listing of Example Programs of Section 4}

The first program $P_{1}(i)$, parameterized by $i \in \mathbb{N}$, has a single Boolean global variable $\mathrm{b}, i$ procedures named $p_{1}, \ldots, p_{i}$, which assert $\mathrm{b}$ to be false and set $\mathrm{b}$ 
$\mathrm{b}$ to be false, sets $\mathrm{b}$ to true, and posts $p_{2}$, while $p_{2}$ sets $\mathrm{b}$ to false and posts $p_{1}$. Initially, the program $P_{2}$ sets $\mathrm{b}$ to false and posts a single $p_{1}$ task. Again here, since a $p_{2}$ task must execute between each $p_{1}$ task, each of the assertions are guaranteed to hold.

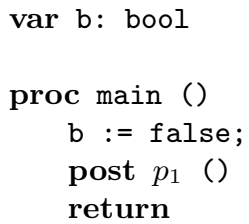

var b: bool

proc main ()

$\mathrm{b}:=$ false;

post $p_{1}$ ()

return

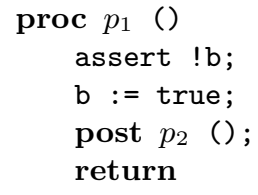

Figure 6p compares the time required to verify $P_{2}$ for various bounds $n$ on the number of tasks explored. Note that although every execution of $P_{2}$ uses only size 1 task-queues, to explore all $n$ tasks in any given execution, the number of phases must be at least $n$, since each task must execute in its own phase.

\section{The Multi-Processor Breadth-First Scheduler}

Here we define a deterministic, non-blocking, delay-accessible delaying scheduler $M_{\text {bfs }}$ which though perhaps odd from an operational point of view, has a very useful application: given a multi-processor message-passing program $P$, the phaseand delay-bounded executions of $P$ according to $M_{\mathrm{bfs}}$ are simulated by executions of a sequential program $P^{\prime}$; furthermore, $P^{\prime}$ is obtained by a simple code-to-code translation of $P$ which does not explicitly represent pending-task queues.

Let $U$ be a set of identifiers uniquely identifying each task along an execution with a single initially-pending task $u_{0} \in U$. Our scheduler keeps a monotonically increasing phase number $i \in \mathbb{N}$, along with an ordered task-posting tree $T$ over nodes $U$, a completion-labeling $\sqrt{ }: U \rightarrow \mathbb{B}$, and a phase-labeling $\Phi: U \rightarrow \mathbb{N}$. Initially the tree contains a single node $u_{0}$, with $\Phi\left(u_{0}\right)=0$ and $\sqrt{ }\left(u_{0}\right)=$ false. As additional tasks are posted, we add them as children of the posting task, in the order they are posted. Normally, the scheduler allows tasks to execute to completion; when a task does complete, the scheduler marks it as completed. When choosing the next task to execute, our scheduler selects the smallest - in breadth-first order over the task-posting tree-unexecuted task in the current phase; if there are no non-completed tasks in the current phase, the scheduler moves to the next phase. In this way, the scheduler executes all tasks in phase order, and same-phase tasks in breadth-first order of the task-posting tree.

To implement delaying, our scheduler also keeps a phase-delay counter $\Delta(\rho)$ : $\mathbb{N}$ for each processor $\rho$. Supposing an executing task $u$ has phase- $i$ on a processor whose phase-delay counter has current value $j$, the task $u$ is treated as though it is in phase $i+j$. When a processor is delayed, its phase-delay counter is simply incremented; the effect is to shift all following tasks on the given processor one additional phase later. Delaying causes the currently executing task to be interrupted and resumed in the following phase. 
Formally the Breadth-First Scheduler $M_{\mathrm{bfs}}=\langle D$, empty, enabled, step, delay $\rangle$ is defined over scheduler objects $m=\langle i, T, \sqrt{ }, \Phi, \Delta\rangle \in D$ as described above; the initial object is empty $=\left\langle 0, T_{0}, \sqrt{ }_{0}, \Phi_{0}, \Delta_{0}\right\rangle$, where $T_{0}$ is the single-node tree with root $u_{0}, \sqrt{ }_{0}(u)=$ false and $\Phi_{0}(u)=0$ for all $u \in U$, and $\Delta_{0}(\rho)=0$ for all $\rho \in$ Pids. The enabled $(\langle i, T, \sqrt{ }, \Phi, \Delta\rangle, \xi)$ operation uniquely returns the processor identifier $\rho$ of the smallest task $u$-according to the breadth-first order of $T$ - such that $\Phi(u)+\Delta(\rho)=i$. The $\operatorname{step}\left(\left\langle i_{1}, T_{1}, \sqrt{ }_{1}, \Phi_{1}, \Delta\right\rangle, \xi_{1}, \xi_{2}\right)$ operation for a transition $\tau=\xi_{1}(\rho) \rightarrow{ }^{\mathrm{S}} \xi_{2}(\rho)$ returns $\left\langle i_{2}, T_{2}, \sqrt{ }_{2}, \Phi_{2}, \Delta\right\rangle$ such that

- If $\tau$ is a COMPLETE-step of task $u$, then $\sqrt{ }_{2}=\sqrt{ }_{1}(u \mapsto$ true $)$; otherwise $\sqrt{2}=\sqrt{ }_{1}$.

- If $\tau$ is a POST- or Self-Post-step of task $u$ posting task $u^{\prime}$, then $T_{2}$ is obtained from $T_{1}$ by adding to $u$ new a rightmost child $u^{\prime}$, and $\Phi_{2}=\Phi_{1}\left(u^{\prime} \mapsto\right.$ $\left.\Phi_{1}(u)+\Delta(u)\right)$; otherwise, $T_{2}=T_{1}$ and $\Phi_{2}=\Phi_{1}$.

- If there no longer exists a non-completed task $u$ on some processor $\rho^{\prime}$ such that $\Phi_{2}(u)+\Delta\left(\rho^{\prime}\right)=i_{1}$ then $i_{2}=i_{1}+1$; otherwise $i_{2}=i_{1}$.

The delay $\left(\left\langle i_{1}, T, \sqrt{ }, \Phi, \Delta_{1}\right\rangle, \rho, \xi\right)$ operation returns $\left\langle i_{2}, T, \sqrt{ }, \Phi, \Delta_{2}\right\rangle$ such that

- $\Delta_{2}=\Delta_{1}\left(\rho \mapsto \Delta_{1}(\rho)+1\right)$ increments $\Delta_{1}$ 's mapping for processor $\rho$.

- If there no longer exists a non-completed task $u$ on some processor $\rho^{\prime}$ such that $\Phi(u)+\Delta_{2}\left(\rho^{\prime}\right)=i_{1}$, then $i_{2}=i_{1}+1$; otherwise $i_{2}=i_{1}$.

According to our definition, $M_{\mathrm{bfs}}$ repeatedly picks a unique processor $\rho$ to execute such that $\rho$ is non-idle or has pending tasks, and $\rho$ 's first non-idle or pending task $u$ has the lowest offsetted phase $\Phi(u)+\Delta(\rho)$ of any task on any processor.

Note that $M_{\mathrm{bfs}}$ is a deterministic delaying scheduler which executes all tasks of a given phase before any task of a subsequent phase. Since $M_{\mathrm{bfs}}$ must pick an enabled task so long as there are pending tasks on some processor, $M_{\mathrm{bfs}}$ is non-blocking. Finally, since for any $i=\Phi(u)+\Delta(\rho)$, repeatedly delaying every other processor $\rho^{\prime} \neq \rho$ eventually increments $\Delta\left(\rho^{\prime}\right)$ such that for any pending $u^{\prime}$ on $\rho^{\prime}, \Phi\left(u^{\prime}\right)+\Delta\left(\rho^{\prime}\right)>i, M_{\mathrm{bfs}}$ is delay accessible.

On non-delaying executions, $M_{\mathrm{bfs}}$ essentially performs a phase-by-phase breadth-first traversal of the task-posting tree $T$ - a tree which includes tasks across all processors. Interestingly and essentially for our sequential encoding of $M_{\mathrm{bfs}}$ in Section 5, on a per-phase basis, with respect to any individual processor, the breadth-first traversal of the task-posting tree is identical to depth-first traversal. This follows from the fact that no task may have a same-processor ancestor in the same phase, and that processors do not share memory.

\section{E Proofs to Selected Theorems}

Theorem 3. The k-phase bounded state-reachability problem is undecidable for multi-processor programs with finite data domains.

Proof. We proceed by reduction from Post's correspondence problem [19]: given words $\alpha_{1}, \ldots, \alpha_{n}, \beta_{1}, \ldots, \beta_{n} \in \Sigma^{*}$ of a finite alphabet $\Sigma$ such that $|\Sigma| \geq 2$, find 
